\title{
Analysis of a Prey-Predator Fishing Model for Harvesting with The Limited Effect of Density and Toxicity
}

\author{
Dita Anies Munawwaroh ${ }^{1, *}$, Sutimin ${ }^{1}$, and Siti Khabibah ${ }^{1}$ \\ ${ }^{1}$ Departement of Mathematics, Faculty of Sciences and Mathematics, Universitas Diponegoro, Semarang, Indonesia
}

\begin{abstract}
We proposed a model of prey-predator fishing by considering proportion of prey density and toxicity in ecosystem. The model is analysed to study about biological equilibrium, bionomic equilibrium and its stability. The aim of the model is to determine the optimal sustainable harvesting for each species. The optimal harvesting is resulted from Pontryagin's maximal principle. The global stability of coexistence equilibrium is analyzed from Lyapunov function. The effect of toxicity leads to the decreasing of sustainable harvesting.
\end{abstract}

Keyword: Prey-predator; harvesting; equilibrium; stability

\section{Introduction}

Indonesia has diversity in freshwater ecosystems, but lately it goes extinction because of red devil fish (Amphilophus Labiatus). The red devil as predator came from Nicaraguan Lakes through cultivation of the domestic fish $[1,2]$.

In other condition, the ecosystem get problems from effect of toxicants that come from industries, household waste etc. leading the effect for the freshwater ecosystems Hallam [3-9] . The ecological effects of toxicants released by the marine biota was studied by Tapasi [10].

The harvesting of fish was introduced by Smith [11] in a mathematical modelling. The researcher seen that the harvesting of fish must fulfil the optimal condition in so that it was not disrupting the ecosystem. Smith modelled competing two specie competitive in an ecosystem, to obtain optimal problem used Pontryagin's maximal principle [12].

In addition, an ecological model about two preys and one predator species is proposed by Sutimin et.al. [13]. The model considered the competition two preys with one predator. Functional response of species are assumed in bilinear term.

In this paper, we discuss a model by considering the effect of toxin and limited prey density. We analyse the existence of the equilibrium and its stability for the model including nontrivial equilibrium. We will also examine about bionomic equilibrium and optimal problem in harvesting.

\section{Model of mathematics}

First, we assume that the ecosystems is freshwater, with just two species that will be a prey, and a predator for the other.A prey will be said $x(t)$, and a predator will be said $y(t)$. Then, we assume the freshwater get pollution so may cause toxin for the two species that have limited density to live. The model is given by the following,

$$
\begin{aligned}
& \frac{d x}{d t}=r_{1} y\left(1-\left(\frac{x}{K_{1}}\right)\right)-\alpha_{12}\left(\frac{x y}{x+y}\right)-q_{1} E x-\beta_{2} y \\
& \frac{d y}{d t}=r_{2} y\left(1-\left(\frac{y}{K_{2}}\right)\right)-\alpha_{21}\left(\frac{x y}{x+y}\right)-q_{2} E y-\beta_{2} y
\end{aligned}
$$

where $r_{1}, r_{2}$ are constant growth rates of prey and predator populations with $K_{1}$ and $K_{2}$ the carrying capacities $K_{1}$ and $K_{2}$, respectively. The prey population decreases due to predator effect with the rate $\alpha_{12}$, conversely, predator population increases with the rate $\alpha_{21}$ supported by prey consumption. We assume that the functional response is in term of $\frac{x}{x+y}$, and $\beta_{1}, \beta_{2}$ are death rates of prey and predator due to toxin, catchability coefficients of prey and predator biomass are denoted $q_{1}, q_{2}$ and $\mathrm{E}$ is the harvesting effort unit of prey and predator biomass.

\section{Analysis of system}

We discuss about equilibrium states, then analyse them for its stability and the bionomic solution for the model.

\subsection{Existence of equilibrium points}

There are equilibrium states that are given by the following, $S_{0}(0,0)$, the state of extinction for two species of fish. Then, $S_{2}\left(x^{*}, 0\right)$, the state prey species exists, but predator dies, where

* Corresponding author: dita.anies@undip.ac.id 
$x^{*}=\frac{K_{1}\left(r_{1}-q_{1} E-\beta_{1}\right)}{r_{1}} . \quad r_{1}>q_{1} E+\beta_{1}$. Next, the state $P_{2}\left(0, y^{*}\right)$ : predator survives but prey extinct, with $y_{1}^{*}=\frac{K_{2}\left(r_{2}-q_{2} E-\beta_{2}\right)}{r_{2}}$ for $r_{2}>q_{2} E+\beta_{2}$. he last, the state of $P_{3}\left(x^{*}, y^{*}\right)$ is the state when the prey and predator exist, where $x^{*}$ is the solution of polynomial

$a_{2} x^{2}+a_{1} x+a_{0}=0$

where

$$
\begin{aligned}
& a_{2}= r_{1}\left(\alpha_{21} K_{1} r_{1}+\alpha_{12} K_{1} r_{2}\right) \\
& a_{1}= K_{1}\left(-r_{2} \alpha_{12} K_{1} r_{1}+r_{2} \alpha_{12} K_{1} q_{1} E+r_{2} \alpha_{12} K_{1} \beta_{1}+\right. \\
& r_{2} \alpha_{12} K_{2} r_{1}-\beta_{2} \alpha_{12} K_{2} r_{1}+2 \alpha_{21} K_{2} r_{1} q_{1} E+ \\
& 2 \alpha_{21} K_{2} r_{1} \alpha_{12}-2 \alpha_{21} K_{2} r_{1}^{2}-q_{2} E \alpha_{12} K_{2} r_{1}+ \\
&\left.2 \alpha_{21} K_{2} r_{1} \beta_{1}\right) \\
& a_{0}=K_{1}^{2} K_{2}\left(-r_{1}+\beta_{1}+\alpha_{12}+q_{1} E\right)\left(E \alpha_{21} q_{1}-\right. \\
& \quad E q_{2} \alpha_{12}+\alpha_{12} \alpha_{21}+r_{2} \alpha_{12}+\beta_{1} \alpha_{21}-\beta_{2} \alpha_{12}- \\
&\left.\quad r_{1} \alpha_{21}\right)
\end{aligned}
$$

The polynomial has exactly one positive root of $x^{*}$, when $E \alpha_{21} q_{1}-E q_{2} \alpha_{12}+\alpha_{12} \alpha_{21}+r_{2} \alpha_{12}+\beta_{1} \alpha_{21}-$ $\beta_{2} \alpha_{12}-r_{1} \alpha_{21}>0$.

\subsection{Analysis of nontrivial equilibrium point}

The following theorem give that non trivial solution is the the global stable for the model.

Theorem 3.1. The point $P_{3}\left(x^{*}, y^{*}\right)$ is global stable if, $y^{*}>y$.

Proof: Lyapunov function is defined

$$
\begin{gathered}
V(x, y)=\left[\left(x-x^{*}\right)-x^{*} \log \left(\frac{x}{x^{*}}\right)\right]+h\left[\left(y-y^{*}\right)-\right. \\
\left.y^{*} \log \left(\frac{y}{y^{*}}\right)\right]
\end{gathered}
$$

We have the derivative of $V$ to $t$ for the system (1),

$$
\frac{d V}{d t}=\frac{x-x^{*}}{x} \frac{d x}{d t}+h\left(\frac{y-y^{*}}{y}\right) \frac{d y}{d t}
$$

by substituting the system (1) to above equation, we get

$$
\begin{aligned}
& \frac{d V}{d t}=\left(x-x^{*}\right)\left(-\frac{\alpha_{12} y}{x+y}+\frac{\alpha_{12} y^{*}}{x^{*}+y^{*}}\right)+h\left(y-y^{*}\right)\left(-\frac{\alpha_{21} x}{x+y}+\right. \\
& \left.\frac{\alpha_{21} x^{*}}{x^{*}+y}\right)+\frac{h\left(y-y^{*}\right)\left(-r_{2} y+r_{2} y^{*}\right)}{K_{2}}+\frac{\left(x-x^{*}\right)\left(-r_{1} x+r_{1} x^{*}\right)}{K_{1}}
\end{aligned}
$$

From $y^{*}>y$, we can conclude that $\frac{d V}{d t}<0$, it completes the proof.

\subsection{Bionomic steady state}

The condition of bionomic is obtained by equity of total revenue and effort for harvesting. Let $C$ is defined as the fishing cost per effort unit, $p_{1}, p_{2}$ are the prices per unit for prey and predator, respectively. The economic value is in following

$$
\pi(x, y, E)=\left(p_{1} q_{1} x+p_{2} q_{2} y-C\right) \mathrm{E}
$$

From the system (1), we must get, so it will be

$$
\begin{gathered}
\frac{d x}{d t}=0 \\
r_{1}\left(1-\left(\frac{x}{K_{1}}\right)\right)-\alpha_{12}\left(\frac{y}{x+y}\right)-q_{1} E-\beta_{1}=0 \\
E=\frac{1}{q_{1}}\left[r_{1}\left(1-\left(\frac{x}{K_{1}}\right)\right)-\alpha_{12}\left(\frac{y}{x+y}\right)-\beta_{1}\right] .
\end{gathered}
$$

Then,

$$
\begin{gathered}
\frac{d y}{d t}=0 ; \\
r_{2}\left(1-\left(\frac{y}{K_{2}}\right)\right)-\alpha_{21}\left(\frac{x}{x+y}\right)-q_{2} E-\beta_{2}=0 ; \\
E=\frac{1}{q_{2}}\left[r_{2}\left(1-\left(\frac{y}{K_{2}}\right)\right)-\alpha_{21}\left(\frac{x}{x+y}\right)-\beta_{2}\right] .
\end{gathered}
$$

By eliminating $E$ in Equation (5), (6), and (7), and substituting $y$ from Equation (5) to Equation (6) and (7), we get the solution of $x$ from the equation

$$
A_{2} x^{2}+A_{1} x+A_{0}=0
$$

$A_{2}=\frac{r_{2} q_{1} p_{1}}{p_{2} q_{2}^{2} K_{2}}-\frac{r_{2} K_{1} q_{1}-q_{2} K_{2} r_{1}}{q_{1} q_{2} K_{2} K_{1}}-\frac{q_{2} p_{2} r_{1}}{K_{1} q_{1}^{2} p_{1}}$

$A_{1}=\frac{1}{p_{2} q_{2}^{2} K_{2} K_{1} q_{1}^{2} p_{1}}\left(-r_{1} K_{1} q_{1} p_{2}^{2} q_{2}^{2} K_{2}+\right.$

$r_{2} K_{1} q_{1}^{2} p_{2} q_{2} K_{2} p_{1}-2 r_{2} K_{1} q_{1}^{2} p_{1} C+r_{2} K_{1} q_{1}^{2} C p_{2} q_{2}+$

$\alpha_{12} p_{2} q_{2} K_{2} p_{1} K_{1} q_{1}^{2}+\alpha_{12} p_{2} q_{2}^{2} K_{2} p_{1} K_{1} q_{1}-$

$p_{2} q_{2}^{2} K_{2} r_{1} C+p_{2}^{2} q_{2}^{3} K_{2} r_{1} K_{1}-p_{2} q_{2}^{2} K_{2} p_{1} K_{1} q_{1} r_{1}-$ $p_{2}^{2} q_{2}^{3} K_{2} \beta_{1} K_{1}+p_{2} q_{2}^{2} K_{2} p_{1} K_{1} q_{1} \beta_{1}+p_{2}^{2} q_{2}^{2} K_{2} K_{1} q_{1} \beta_{2}-$ $\left.p_{2} q_{2} K_{2} K_{1} q_{1}^{2} \beta_{2}\right)$

$A_{0}=q_{2}^{2} p_{2} K_{2} r_{1} K_{1} C+\left(-K_{1} q_{1} p_{2} q_{2} K_{2} C+K_{1} q_{1} C^{2}\right) r_{2}-$ $q_{2}^{2} p_{2} K_{2} \beta_{2} K_{1} C+\beta_{2} p_{2} q_{2} K_{2} K_{1} q_{1} C-$

$\alpha_{21} p_{2} q_{2} K_{2} K_{1} q_{1} C-q_{2}^{2} p_{2} K_{2} \alpha_{12}$

From the equation (8), we can see that sum of roots is $\frac{-A_{1}}{A_{2}}$ and its product is $\frac{A_{0}}{A_{2}}$. The equation (8) has only one positive root if $A_{2}>0$ and $A_{0}<0$. So, we must restrict

$$
\frac{q_{2} K_{2} r_{1}-r_{2} K_{1} q_{1}}{q_{1} q_{2} K_{2} K_{1}}>\frac{q_{2} p_{2} r_{1}}{K_{1} q_{1}^{2} p_{1}}
$$

And

$$
\begin{aligned}
K_{1} q_{1} p_{2} q_{2} K_{2} C r_{2} & +q_{2}^{2} p_{2} K_{2} \beta_{2} K_{1}+p_{2} q_{2} K_{2} K_{1} q_{1} C \\
& +q_{2}^{2} p_{2} K_{2} \alpha_{12} K_{1} C>0
\end{aligned}
$$

The bionomic solution is $y_{\infty}=\frac{C-p_{1} q_{1} x_{\infty}}{p_{2} q_{2}}$, so $x_{\infty}=\frac{C}{p_{1} q_{1}}$. The bionomic point $\left(x_{\infty}, y_{\infty}\right)$, with $x_{\infty}<\frac{C}{p_{1} q_{1}}$ is unique if $A_{2}>0$ and $A_{0}<0$.

\subsection{Optimal Harvesting Analysis}

Let $J$ is a continuous function for the revenue $J=\int_{0}^{\infty} \pi(x, y, E, t) e^{-\delta t} d t$

With $\pi(x, y, E, t)$ is economic value, and $\delta$ is discount annual rate. In the problem is to maximize the objective function (9) subject to the system (1). We must control $E(t)$ subject to $0 \leq E(t) \leq E_{\max }$. Hamiltonian function $H$ is given by

$H=\pi(x, y, E)=\left(p_{1} c_{1} x+p_{2} c_{2} y-C\right) E e^{-\delta t}+$
$\lambda_{1}\left[r_{1} x\left(1-\left(\frac{x}{k_{1}}\right)\right)-\alpha_{12}\left(\frac{x y}{x+y}\right)-q_{1} E x-\beta_{1} x\right]+$
$\lambda_{2}\left[r_{2} y\left(1-\left(\frac{y}{k_{2}}\right)\right)-\alpha_{21}\left(\frac{x y}{x+y}\right)-q_{2} E y-\beta_{2} y\right]$

where $\lambda_{1}, \lambda_{2}$ are the adjoint variables. To get an optimal equilibrium solution, we get 
There are

$$
\frac{d \lambda_{1}}{d t}=-\frac{\partial H}{\partial x} \text { and } \frac{d \lambda_{2}}{d t}=-\frac{\partial H}{\partial y} .
$$

$$
\begin{aligned}
& \frac{d \lambda_{1}}{d t}=-p_{1} q_{1} E e^{-\delta t}-\lambda_{1}\left[r_{1}\left(1-\left(\frac{x}{K_{1}}\right)\right)-\frac{r_{1} x}{K_{1}}-\right. \\
& \left.\alpha_{12}\left(\frac{y}{x+y}\right)+\alpha_{12} \frac{x y}{(x+y)^{2}}-q_{1} E-\beta_{1}\right]-\lambda_{2}\left[\left(\frac{\alpha_{21} y}{x+y}\right)-\right. \\
& \left.\frac{\alpha_{21} x y}{(x+y)^{2}}\right]
\end{aligned}
$$

and

$$
\begin{gathered}
\frac{d \lambda_{2}}{d t}=-p_{2} q_{2} E e^{-\delta t}-\lambda_{2}\left[-\frac{a_{12} x}{x+y}+\frac{a_{12} x y}{(x+y)^{2}}\right] \\
\lambda_{1}\left[r_{2}\left(1-\left(\frac{y}{K_{2}}\right)\right)-\frac{r_{2} y}{K_{2}}-\alpha_{21}\left(\frac{x}{x+y}\right)-\alpha_{21} \frac{x y}{(x+y)^{2}}-\right. \\
\left.q_{2} E-\beta_{2}\right] .
\end{gathered}
$$

To obtain the optimal solution of the equilibrium, first step is substitution Equation (7) to Equation (11), and Equation (8) to Equation (12). By eliminating $\lambda_{2}$, we get the differential equation of $\lambda_{1}$,

$\frac{d^{2} \lambda_{1}}{d t^{2}}-P \frac{d \lambda_{1}}{d t}$

$-\frac{\left(Q K_{1} \alpha_{21} y^{2}+P x^{3} r_{1}+2 P x^{2} r_{1} y+P x r_{1} y^{2}-P x \alpha_{12} y K_{1}\right) \lambda_{1}}{K_{1} \alpha_{21} y^{2}}$

$=R e^{-\delta t}$

where

$P=-\frac{r_{2} y}{K_{2}}+\frac{\alpha_{21} x}{x+y}-\frac{\alpha_{21} x y}{x+y}+\frac{\alpha_{21} y}{x+y}$;

$Q=-\frac{\alpha_{12} x}{x+y}+\frac{\alpha_{12} x y}{x+y}$

$R=-\frac{1}{(x+y)^{2}}\left(-p_{1} q_{1} \delta y^{2}-p_{1} q_{1} \delta x^{2}-2 p_{1} q_{1} \delta x y-\right.$

$\left.p_{2} q_{2} \alpha_{21} y^{2}+p_{1} q_{1} P y^{2}+p_{1} q_{1} P x^{2}+2 p_{1} q_{1} P x y\right)$.

We find the differential equation in $\lambda_{1}$

$\frac{d^{2} \lambda_{1}}{d t^{2}}-P \frac{d \lambda_{1}}{d t}-$

$\frac{\left(Q K_{1} \alpha_{21} y^{2}+P x^{3} r_{1}+2 P x^{2} r_{1} y+P x r_{1} y^{2}-P x \alpha_{12} y K_{1}\right) \lambda_{1}}{K_{1} \alpha_{21} y^{2}}=0$.

We see that the sum of roots equal to $P>0$ and $\frac{\left(Q K_{1} \alpha_{21} y^{2}+P x^{3} r_{1}+2 P x^{2} r_{1} y+P x r_{1} y^{2}-P x \alpha_{12} y K_{1}\right)}{K_{1} \alpha_{21} y^{2}}>0$

There are two positive real roots of (13) or complex roots with positive real parts. The solution of (13) is given by

$$
\begin{gathered}
\lambda_{1}(t)=c_{1} e^{\left(0.5 P+0.5 \sqrt{P^{2}-4 Q}\right) t}+c_{2} e^{\left(0.5 P-0.5 \sqrt{P^{2}-4 Q}\right) t} \\
-\frac{R e^{-\delta t}}{Q+P \delta+\delta^{2}}
\end{gathered}
$$

It is clear that $\lambda_{1}(t)$ is bounded for $t \rightarrow \infty$, if only if $c_{1}=$ $c_{2}=0$. Thus we have

where

$$
\lambda_{1}(t)=\frac{R e^{-\delta t}}{Q+P \delta+\delta^{2}}
$$

$R=\frac{1}{(x+y)^{2}}\left(p_{2} q_{2} \delta x^{2}+2 p_{2} q_{2} \delta x y+p_{2} q_{2} \delta y^{2}+\right.$

$\left.p_{1} q_{1} \alpha_{12} x^{2}+p_{2} q_{2} Q x^{2}+2 p_{2} q_{2} Q x y+p_{2} q_{2} Q y^{2}\right)$.

In the similar, it is obtained,

$$
\lambda_{2}(t)=\frac{S e^{-\delta t}}{Q+P \delta+\delta^{2}}
$$

\section{CONCLUSION}

From the result and discussion, we get the conclusion that ecological model of fishery for harvesting with two species as prey-predator, has 4 equilibrium states. For nontrivial equilibrium states was analysed the global stability. The model has unique positive state for bionomic solution. The optimal decision in long term for the sustainable harvesting incorporating toxin in the ecosystem was investigated. The results show that the toxin reduces the productivity of harvesting. Therefore to increase the income for harvesting, it is needed the environmental sustainability with low carbon growth to support sustainable economic growth

\section{Acknowledgements}

This work is partly founded by DIPA (2018) Faculty of Sciences and Mathematics, Universitas Diponegoro, Semarang, Indonesia.

\section{References}

A.K. Sakai, F.W Allendorf, J.S Holt, Lodge D.M, Molofsky J, Kimberly A, S. Baughman, R.J. Cabin, j.e. Cohen, N.C. Ellestrand, D.E. McCauley, P. O’Neil, I.M. Parker, J.N Thompson, S.G. Weller, Annu. Rev.Ecol.Syst., 32 (2001).

2. M. Clavero, Garcy'a-Berthou E. Trends Ecol Evol 20 (2005).

3. T.G. Hallam, C.W. Clark, J. Theor. Biol 93 : 303-311 (1982)

4. T.G. Hallam, T.J. de Luna, Theor. Biol 109 : 411-429 (1984)

5. J. Chattopadhyay, Ecol.Model 84 : 297 (1997)

6. A. Mukhopadhyay, J. Chattopadhyay, P.K. Tapaswi, Math. Biosci 149 :167-189 (1998)

7. B.Dubey, J. Hussain, Ecol.Model 129: 195-207 (2000)

8. T.K. Kar, K.S. Chaudhuri, Ecol.Model 161 : 125-137 (2003)

9. T.K Kar, U.K Pahari, K.S. Chaudhuri, J. Biol. Syst $12: 301-313$ (2004)

10. T. Das, R.N Mukerjee, K.S. Chaudhuri, App.Math.Model 33 : 2282-2292 (2009)

11. J. Maynard Smith, Model in Ecology, Cambridge University Press, 1974 p.146

12. L.S Pontryagin, V.S Boltyanski Gamkrelidze, E. F. Mishchenco, The Mathematical Theory of Optimal Precesses, Wiley, New York, 19881962

13. Sutimin, S. Khabibah, Dita A.M., E3S Web of Confrences 31 : 08008 (2018) 